\title{
Translational Chern-Simons Action and New Planar Particle Dynamics
}

\author{
J. Lukierski \\ Institute for Theoretical Physics, University of Wrocław, \\ pl. Maxa Borna 9, 50-204 Wrocław, Poland \\ email:lukier@ift.uni.wroc.pl \\ P.C. Stichel \\ An der Krebskuhle 21 \\ D-33619 Bielefeld \\ email:pstichel@gmx.de
}

W.J. Zakrzewski

Department of Mathematical Sciences, University of Durham, Durham DH1 3LE, UK email:W.J.Zakrzewski@durham.ac.uk

\begin{abstract}
We consider a nonstandard $D=2+1$ gravity described by a translational Chern-Simons action, and couple it to the nonrelativistic point particles. We fix the asymptotic coordinate transformations in such a way that the space part of the metric becomes asymptotically Euclidean. The residual symmetries are (local in time) translations and rigid rotations. The phase space Hamiltonian $H$ describing two-body interactions satisfies a nonlinear equation $H=\mathcal{H}(\vec{x}, \vec{p} ; H)$ what implies, after quantization, a nonstandard form of the Schrödinger equation with energy-dependent fractional angular momentum eigenvalues. Quantum solutions of the two-body problem are discussed. The bound states with discrete energy levels correspond to a confined classical motion (for the planar distance between two particles $r \leq r_{0}$ ) and the scattering states with continuous energy correspond to classical motion for $r>r_{0}$.
\end{abstract}




\section{Introduction}

The aim of this paper is to study a new type of gravitational point particle interactions in two space dimensions. The classical and quantum dynamics of point sources interacting with standard $(2+1)$-dimensional gravity has been well known since the papers by Staruszkiewicz, Deser, Jackiw and t'Hooft were published (see [1-4]).

In this paper we consider a new system of coupled two-dimensional torsion fields with point particles, derived in the following way:

i) We assume a nonstandard gravitational action described by a translational Chern-Simons term [5-7]

$$
S_{T}=\frac{1}{\lambda} \int d^{3} x \varepsilon^{\mu \nu \rho} E_{\bar{\mu}}^{\underline{\alpha}} T_{\bar{\nu}}^{\underline{\beta}} \eta_{\underline{\alpha} \underline{\beta}},
$$

where $E_{\bar{\mu}}(\bar{\nu}=0,1,2 ; \mu=0,1,2)$ describes $(2+1)$-dimensional dreibeins, and the Abelian strength

$$
T_{\mu} \frac{\rho}{\nu}=\partial_{\mu} E_{\bar{\nu}}^{\frac{\rho}{2}}-\partial_{\nu} E_{\bar{\mu}}^{\frac{\rho}{\mu}}
$$

provides the components of a $(2+1)$-dimensional torsion tensor field. Note that a linear torsion Lagrangian taking the form of a translational Chern-Simons term can be introduced only in $(2+1)$ dimensions.

Here we would like to recall that, following Witten [5], the standard gravitational Einstein action in $(2+1)$ dimensions is described by another Chern-Simons theory

$$
S_{E}=\frac{1}{\lambda} \int d^{3} x \varepsilon^{\mu \nu \rho} E_{\bar{\mu}}^{\alpha} \omega_{\bar{\nu} \rho}^{\underline{\beta}} \eta_{\underline{\alpha} \beta}
$$

where $\omega \frac{\alpha}{\mu \nu}$ are the $O(2,1)$ spin connection components.

ii) In this paper we consider gravitational interactions of nonrelativistic $D=2$ particles described by the trajectory $\vec{x}(t)=\left(x_{1}(t), x_{2}(t)\right)$, which are covariant under the following set of nonrelativistic local transformations:

$$
x_{l}^{\prime}=x_{l}^{\prime}(\vec{x}, t), \quad t^{\prime}=t+a,
$$

which imply that

$$
\dot{x}_{i}^{\prime}=\frac{\partial x_{i}^{\prime}}{\partial x_{j}} \dot{x}_{j}^{\prime}+\frac{\partial x_{i}^{\prime}}{\partial t}, \quad \frac{d t^{\prime}}{d t}=1
$$

The set of nonrelativistic reparametrization-invariant velocities then takes the form $(\underline{a}, \underline{b}=1,2)$ :

$$
\xi^{\underline{a}}=E^{\underline{a}} \dot{x}_{i}+E^{\underline{a}},
$$

which can be obtained from the relativistic reparametrization-invariant velocities 


$$
\dot{x}^{\mu} \rightarrow \xi^{\underline{\mu}}=E^{\underline{\mu}} \dot{x}^{\nu}=\left(\xi^{\underline{0}}, \xi^{\underline{a}}\right),
$$

by putting $x_{0}=t$ and introducing a nonrelativistic gauge fixing condition $E^{\underline{0}}=1$, $E^{\underline{0}}=0$. In such a gauge we obtain the nonrelativistic form of the action (1.1), invariant under (1.4)

$$
S_{T}^{(\mathrm{NR})}=\frac{1}{\lambda} \int d^{3} x e^{\mu \nu \rho} E_{\underline{\mu}}^{\underline{a}} T \frac{a}{\nu \rho}=\frac{1}{\lambda} \int d t d^{2} x\left(e^{\underline{a}} B^{\underline{a}}-\varepsilon_{j k} h_{j}^{\underline{q}} \mathcal{E}_{k}^{\underline{a}}\right),
$$

where

$$
B^{\underline{a}}=\varepsilon_{j k} \partial_{j} h_{k}^{\underline{a}} \quad \mathcal{E}_{k}^{\underline{a}}=\partial_{t} h_{k}^{\underline{a}}-\partial_{k} e^{\underline{\underline{a}}} .
$$

We denote $h \frac{a}{\kappa}=E \frac{a}{\kappa}$ and $e^{\underline{a}}=E_{0}^{\underline{a}}$. The fields $B^{\underline{a}}$ and $\mathcal{E}_{k}^{\underline{a}}$ play the role of the magnetic and electric fields, respectively, with the internal $O(2)$ index $\underline{a}$ describing the $D=2$ nonrelativistic rotation group.

In Sect. 2 we discuss a coupled classical field + point particles system. Firstly, we present general considerations for the $N$-body particle dynamics, and then we consider the two-body problem in classical theory. In this case we obtain, in terms of relative planar coordinates, a new type of dynamics which can be understood in two equivalent ways:

- as a $D=2$ free particle system with nonstandard, symplectic structure

- as a modified $D=2$ free particle system with an energy-dependent angular momentum $\bar{l}(E)$ and a canonical symplectic structure.

We also show that the classical solutions split into two classes for $\lambda \bar{l}<0$

- for the planar particle distances

$$
r<r_{0}=\left(\frac{|\lambda \bar{l}(E)|}{2 \pi}\right)^{\frac{1}{2}}
$$

they describe bounded motion while

- for $r>r_{0}$ they describe the scattering.

In Sect. 3 we introduce the corresponding quantum mechanics, described by a nonstandard Schrödinger equation, and discuss the energy eigenvalues and wave eigenfunctions.

We find that the exact values of the energy levels for the confined motion $\left(r<r_{0}\right)$ can be determined only numerically.

The final remarks, concerning possible physical interpretations of our solutions are presented in the last, fourth Section.

It should be added that the present model is a generalization of the $(1+1)$ dimensional model, presented by one of the present authors (PCS) in $[8,9]$. 


\section{New Planar Particle Dynamics: Classical The- ory}

\section{$2.1 \quad$ N-Particle System}

Let us discuss, in some detail, a set of coupled equations describing an interacting two-dimensional particle - torsion field system, with the torsion fields being given by the Abelian Chern-Simons action (1.3). Introducing $N$ trajectories $\vec{x}_{\alpha}(t)=\left\{x_{\alpha}^{i}(t)\right\}$ $(i=1,2 ; \alpha=1, \ldots N)$ for $N$ particles and the notation

$$
E_{\bar{\mu} ; \alpha}^{a}(t) \equiv E_{\bar{\mu}}^{a}\left(\vec{x}_{\alpha}(t), t\right)
$$

we find that the action for $N$ particles in $D=2$ dimensions, in the first order formalism, can be written as

$$
S_{\text {part }}^{(N)}=-\sum_{\alpha=1}^{N} m_{\alpha} \int d t\left[\frac{1}{2} \xi \frac{a}{\alpha} \xi \frac{a}{\alpha}-\xi \frac{a}{\alpha}\left(E_{j ; \alpha}^{\underline{a}} \dot{x}_{\alpha}^{j}+E_{0, \alpha}^{\underline{a}}\right)\right],
$$

thus providing the constraint formula

$$
\xi \frac{a}{\alpha}=E_{j ; \alpha}^{a} \dot{x}^{j}+E_{0, \alpha}^{\underline{a}} .
$$

We can now derive the equations describing the $D=2$ coupled particle-torsion field system, described by the action $S=S_{\text {part }}^{(N)}+S_{\mathrm{T}}^{(\mathrm{NR})}$ (see (1.8) and (2.2)). They take the form:

$$
\begin{gathered}
\dot{\xi} \frac{a}{\alpha} \cdot E_{i, \alpha}^{\underline{a}}+\xi \frac{a}{\alpha} \cdot T_{\mu i, \alpha}^{a} \dot{x}^{\mu, \alpha}=0 \\
T_{\mu \nu}^{a}=\partial_{\mu} E_{\nu}^{a}-\partial_{\nu} E_{\mu}^{a}=-\frac{\lambda}{2} \epsilon_{\mu \nu \rho} \sum_{\beta} \xi_{\beta}^{\frac{a}{\beta}} \dot{x}^{\rho, \beta} \delta\left(\vec{x}-\vec{x}^{\beta}\right) .
\end{gathered}
$$

The second term in (2.4), due to the antisymmetry of the $\epsilon$-tensor in (2.5), can be rewritten as

$$
\xi \frac{a}{\alpha} \cdot T_{\mu i, \alpha}^{\underline{a}} \dot{x}^{\mu, \alpha}=-\frac{\lambda}{2} \epsilon_{\mu i \rho} \sum_{\substack{\beta \\ \beta \neq \alpha}} \xi \frac{a}{\beta} \xi \frac{a}{\alpha} \dot{x}^{\rho, \beta} \dot{x}^{\mu, \alpha} \delta\left(\vec{x}_{\alpha}-\vec{x}_{\beta}\right),
$$

which is infinite for coinciding particle positions and vanishes otherwise. If we restrict our configuration space only to noncoinciding particle positions we have

$$
\dot{\xi} \frac{a}{\alpha} \cdot E \frac{a}{\alpha ; i}=0
$$

which leads, for points in the configuration space where the metric is non-degenerate, to

$$
\dot{\xi} \frac{a}{\alpha}=0
$$


Let us now consider the field equation (2.5). Its general solution can be written in the pure gauge form

$$
E_{\bar{\mu}}^{\underline{a}}(\vec{x}, t)=\widetilde{E}_{\bar{\mu}}^{a}(\vec{x}, t)+\partial_{\mu} \Lambda^{\underline{a}}(\vec{x}, t)=\partial_{\mu} \widetilde{\Lambda}^{\underline{a}}(\vec{x}, t),
$$

where

$$
\widetilde{E}_{\bar{\mu}}^{\underline{a}}(\vec{x}, t):=-\frac{\lambda}{4 \pi} \partial_{\mu} \sum_{\alpha} \xi_{\alpha}^{\underline{a}} \Phi\left(\vec{x}-\vec{x}_{\alpha}\right)
$$

and

- $\Lambda \underline{a}$ is an $O(2)$-vector valued pair of regular gauge functions,

- $\Phi(\vec{x})$ is a singular gauge function satisfying the following equation (see e.g. [10]):

$$
\epsilon^{i j} \partial_{i} \partial_{j} \Phi(\vec{x})=2 \pi \delta(\vec{x}) .
$$

As a solution of (2.11) we can take

$$
\Phi(\vec{x})=\arctan \frac{x_{2}}{x_{1}}
$$

i.e.

$$
\partial_{k} \Phi(x)=-\varepsilon_{k l} \partial_{l} \ln |\vec{x}| .
$$

where (2.13) has to be regularized in such a way that it is well defined everywhere and therefore vanishes for $\vec{x} \rightarrow 0$ (see e.g. [11]).

Let us note that the solutions for the fields $E_{\bar{\mu}}(\vec{x}, t)$ with asymptotically nonvanishing gauge function $\Lambda^{a}$ are not solutions of the Hamilton's variational principle for the action $S$. The bad asymptotic (as $r \rightarrow \infty$ ) behaviour of $E_{\bar{\mu}}(\vec{x}, t)$ leads to the appearance of nonvanishing surface integrals and, in consequence, our $E_{\vec{\mu}}(\vec{x}, t)$ do not minimize the action. In order to take into consideration the particular asymptotic behaviour of $\Lambda^{a}$ we fix our gauge as follows:

$$
\Lambda^{i}(\vec{x}, t)=x^{i}-a^{i}(t)
$$

and modify the field Lagrangian by adding two surface integrals]

$$
\begin{aligned}
& I_{1}=\frac{1}{\lambda} \int d^{2} x v^{\underline{\mathrm{a}}} \cdot \epsilon^{i j} \partial_{i} \widetilde{E}_{j}^{a}(\vec{x}, t) \\
& I_{2}=\frac{1}{\lambda} \int d^{2} x \epsilon^{i j} \partial_{i} \widetilde{E}_{0}^{j}
\end{aligned}
$$

where we have defined

$$
v^{\underline{a}}(t):=\dot{a}^{\underline{a}}(t) .
$$

Notice that with the gauge (2.14) the space part of our metric becomes asymptotically Euclidean. Due to the asymptotic behaviour

$$
\widetilde{E}_{\bar{\mu}}^{a}(\vec{x}, t) \rightarrow O\left(r^{-1}\right) \quad \text { as } \quad r \rightarrow \infty
$$

\footnotetext{
${ }^{1}$ see [12] for such a procedure.
} 
the variations of the $\widetilde{E}_{\bar{\mu}}^{a}$ and of the $v^{\underline{a}}$ are independent of each other i.e. the fields $\widetilde{E}_{\bar{\mu}}^{\underline{a}}$ and $v^{\underline{a}}$ can be introduced as new variables. The particle action (2.2) takes the form

$$
S_{\text {part }}^{(\mathrm{N})}[x, \xi, E]=S_{\text {part }}^{(\mathrm{N})}[x, \xi, \widetilde{E}]+\int d t\left[\sum_{\alpha} \xi \frac{a}{\alpha} \cdot \dot{x}_{\alpha}^{\underline{a}}-v^{\underline{a}} \cdot \sum_{\alpha} \xi \frac{a}{\alpha}\right] .
$$

where as the modified field action $S_{\text {field }}(\widetilde{E})$ is given by $S_{T}^{(N R)}$ (eq. (1.8)) but taken as a function of the $\widetilde{E}$ now

$$
S_{\text {field }}(\widetilde{E})=S_{T}^{(N R)}(\widetilde{E})
$$

We see that in the action (2.19) we have separated the variables describing the "bulk" $\left(\widetilde{E}_{\mu}^{a}\right)$ and asymptotic behaviour $\left(v^{a}\right)$. In technical terms, no surface integrals remain when we calculate the functional derivative of the action (2.19) with respect to the $\widetilde{E}$. By varying $S$ with respect to $v^{\underline{a}}$ we obtain the constraint

$$
\sum_{\alpha} \xi \frac{a}{\alpha}=0
$$

The choice (2.14) for $\Lambda^{i}$ breaks asymptotically the invariance with respect to local space translations (1.4). However, as under general coordinate transformations the functions $\widetilde{\Lambda}^{i}$ are scalars, the changes $\delta \widetilde{\Lambda}^{i}$ under (1.4) of both the singular and regular parts of $\widetilde{\Lambda}^{i}$ must vanish and we obtain

$$
\delta x^{i}=\delta a^{i}(t)
$$

where $x^{i}$ as well as $a^{i}$ transform as vectors under rotations in tangent space. Therefore as a residual symmetry we get translations, local in time, and rigid rotations.

Let us calculate the corresponding generator of constant translations (2.21). We find by using Noether's theorem

$$
\begin{aligned}
P^{i} & =\sum_{\alpha} p_{\alpha}^{i}+\frac{2}{\lambda} \int d^{2} x B^{\underline{a}} \widetilde{E}_{i}^{\underline{a}} \\
& =P_{\text {part }}^{i}+P_{\text {field }}^{i},
\end{aligned}
$$

where the canonical particle momenta are obtained from (2.2), (2.9) and (2.14) as

$$
p_{\alpha}^{j}=\xi_{\alpha}^{j}-\frac{\lambda}{4 \pi} \sum_{\substack{\beta \\ \beta \neq \alpha}}\left(\xi \frac{a}{\alpha} \cdot \xi \frac{a}{\beta}\right) \partial_{j} \Phi\left(\vec{x}_{\alpha \beta}\right),
$$

where $\vec{x}_{\alpha \beta}=\vec{x}_{\alpha}-\vec{x}_{\beta}$.

Inserting the explicit expressions for $B^{\underline{a}}$ and $\widetilde{E}_{i}^{a}$ into $(2.22)$ we obtain

$$
P_{\text {field }}^{i}=0 \text {. }
$$

Furthermore, from (2.23) we get

$$
P_{\mathrm{part}}^{i}=\sum_{\alpha} \xi_{\alpha}^{i}
$$


The formula (2.25) describes the linear momentum of the CM-motion for the Nparticle system which vanishes due to the constraint (2.20)2. The same result has been obtained by one of the present authors (PCS) in the one-dimensional case [ 8], [9].

Next we pass to the Hamiltonian formulation. Applying the Legendre transformation to the Lagrangian (2.19) and using the constraint

$$
B^{\underline{a}}=\epsilon_{i j} \partial_{j} h_{i}^{\underline{a}}=-\frac{\lambda}{2} \sum_{\alpha} \xi \frac{a}{\alpha} \delta^{(2)}\left(x-x_{\alpha}\right)
$$

given by the components $\mu=j, \nu=i$ of (2.5) we find the N-particle Hamiltonian to be given by

$$
H^{(\mathrm{N})}=\frac{1}{2} \sum_{\alpha} \xi_{\alpha}^{\underline{a}} \xi^{\underline{a}}+v^{\underline{a}} \cdot \sum_{\alpha} \xi_{\alpha}^{\underline{a}}
$$

\subsection{Hamiltonian Formulation for the Two-Body Problem}

Let us consider now more explicitly the $N=2$ case.

We define:

$$
\xi^{\underline{a}}:=\frac{1}{2}\left(\xi^{\underline{a}}-\xi^{\underline{a}}\right), \quad x^{\underline{a}}:=x_{1}^{\underline{a}}-x^{\underline{a}}, \quad p^{\underline{a}}:=\frac{1}{2}\left(p_{1}^{\underline{a}}-p^{\underline{a}}\right) .
$$

Then using the constraint (2.20) we get from (2.27)

$$
H^{(2)}=\xi^{\underline{a}} \cdot \xi^{\underline{a}}
$$

and

$$
p_{i}=\xi_{i}+\frac{\lambda}{4 \pi}\left(\xi^{\underline{a}} \cdot \xi^{\underline{a}}\right) \partial_{i} \Phi(\vec{x}) .
$$

The Hamiltonian equations take the form

$$
\begin{gathered}
\dot{x}^{i}=\frac{\partial H}{\partial p^{i}}=2\left(\xi^{\underline{a}} \cdot \frac{\partial \xi^{\underline{a}}}{\partial p^{i}}\right), \\
\dot{p}_{i}=-\frac{\partial H}{\partial x_{i}}=-2\left(\xi^{a} \cdot \frac{\partial \xi^{a}}{\partial x^{i}}\right) .
\end{gathered}
$$

Using (2.30) we have

$$
\xi^{\underline{a}} \cdot \frac{\partial \xi^{\underline{a}}}{\partial p^{i}}=\frac{\xi_{i}}{1+\frac{\lambda}{2 \pi}\left(\xi^{\underline{a}} \cdot \partial_{a} \Phi\right)}
$$

\footnotetext{
${ }^{2}$ Analogous situation has been observed in (2+1)-dimensional gravity [13].
} 


$$
\xi^{a} \cdot \frac{\partial \xi^{a}}{\partial x^{j}}=-\frac{\lambda}{4 \pi} \frac{\left(\xi_{a} \xi^{a}\right) \xi^{i} \partial_{i} \partial_{j} \Phi}{1+\frac{\lambda}{2 \pi}\left(\xi^{a} \cdot \partial_{a} \Phi\right)}
$$

Taking the time derivative of (2.30) and using (2.31-32) we get

$$
\dot{\xi}_{i}^{a}+\frac{\lambda}{2 \pi} \partial_{i} \Phi(x) \xi_{j} \dot{\xi}_{j}=0
$$

However, instead of the canonical variables $\left(x_{i}, p_{i}\right)$ we can use the variables $\left(x_{i}, \xi_{i}\right)$. Then the Lagrangian obtained from the Hamiltonian (2.29) would have had the form:

$$
\begin{aligned}
L & =p_{l}\left(\xi_{i}, x_{l}\right) \cdot \dot{x}_{l}-H \\
& =\left(\xi_{l}+\frac{\lambda}{4 \pi} \xi^{2} \partial_{l} \Phi(x)\right) \dot{x}_{l}-\xi^{2} .
\end{aligned}
$$

The variation with respect to $\xi_{i}$ is given by the expression

$$
\xi_{i}=\frac{\frac{1}{2} \dot{x}_{i}}{1-\frac{\lambda}{4 \pi}\left(\dot{x}_{j} \partial_{j} \Phi\right)},
$$

which is equivalent to the Hamiltonian eq. (2.31a) with the insertion of $(2.32 \mathrm{a})$. Inserting (2.35) in (2.34) we get

$$
L=\frac{1}{4} \frac{\dot{x}_{l}^{2}}{1-\frac{\lambda}{4 \pi}\left(\dot{x}_{l} \partial_{l} \Phi\right)} .
$$

In particular if we consider

$$
\begin{aligned}
\operatorname{det}\left(\frac{\partial^{2} L}{\partial \dot{x}_{i} \partial \dot{x}_{j}}\right) & =\frac{1}{4}\left(1-\frac{\lambda}{4 \pi}\left(\dot{x}_{l} \partial_{l} \Phi\right)\right)^{-4} \\
& =\frac{1}{4}\left(1+\frac{\lambda}{2 \pi} \xi_{l} \partial_{l} \Phi\right)^{+4}
\end{aligned}
$$

we see that when the velocities are expressible in terms of the canonical variables then (2.33) gives us

$$
\dot{\xi}_{l}=0 \text {. }
$$

Using the Hamiltonian (2.29) we get for the pair of noncanonical variables the Hamilton equations (2.31a), as $\dot{x}_{l}=\left\{x_{l}, H\right\}$ and (2.38) as $\dot{\xi}_{l}=\left\{\xi_{l}, H\right\}$, if we assume the following nonstandard symplectic structure:

$$
\left\{x^{i}, x^{j}\right\}=\left\{\xi_{i}, \xi_{j}\right\}=0
$$




$$
\left\{x^{i}, \xi_{j}\right\}=\delta^{i}{ }_{j}-\frac{\frac{\lambda}{2 \pi} \xi^{i} \partial_{j} \Phi}{1+\frac{\lambda}{2 \pi}\left(\xi^{a} \partial_{a} \Phi\right)} .
$$

It is easy to check that the Poisson brackets $(2.39 \mathrm{a}-\mathrm{b})$ satisfy the Jacobi identity. For our system we have two conserved angular momenta. If we define (in $D=2$ $\left.\vec{a} \wedge \vec{b}=\epsilon_{i j} a^{i} b^{j}\right)$

$$
l:=\vec{x} \wedge \vec{p}=\vec{x} \wedge \vec{\xi}+\frac{\lambda}{4 \pi} H
$$

we find that $l$ is conserved as well as

$$
\bar{l}:=\vec{x} \wedge \vec{\xi}
$$

because

$$
\frac{d}{d t} \bar{l}=\dot{\vec{x}} \wedge \vec{\xi}=0 .
$$

However, as

$$
\xi^{\underline{a}} \cdot \partial_{a} \Phi=-\vec{\xi} \wedge \vec{\nabla} \ln \left|x^{\underline{c}}\right|=\frac{\bar{l}}{r^{2}},
$$

where $r:=\left|x^{\underline{a}}\right|$, we see that (2.35) can be rewritten as:

$$
\dot{x}^{\underline{a}}=\frac{2 \xi \underline{a}}{1+\frac{\lambda \bar{l}}{2 \pi r^{2}}} .
$$

Note that if $x^{\underline{a}}(0)$ is parallel to $\xi^{\underline{a}}$ we have $\bar{l}=0$ and, in consequence, a free motion on a line.

For $\lambda \bar{l}<0$ it is convenient to introduce the quantity

$$
r_{0}^{2}:=\frac{|\lambda \bar{l}|}{2 \pi}
$$

as then, from the previous results, we see that the relative two-body motion separates in this case into a motion within the interior region given by

$$
r<r_{0}
$$

and a motion within the exterior region given by

$$
r>r_{0} .
$$




\section{New Planar Dynamics: Quantum-Mechanical Two-Body Problem}

The relation (2.30), after the substitution of (2.29), takes the form $\left(H \equiv H^{(2)}\right)$ :

$$
\vec{\xi}=\vec{p}-\frac{\lambda}{4 \pi} H \vec{\nabla} \Phi
$$

Squaring it and using again (2.29) we obtain

$$
H=\vec{p}^{2}-\frac{l^{2}}{r^{2}}+\frac{\bar{l}^{2}}{r^{2}}
$$

where we have used the definition $(2.39 \mathrm{~d})$ of $\bar{l}$.

It should be stressed that

$$
\bar{l}=l-\frac{\lambda}{4 \pi} H
$$

i.e. (3.2a) gives us a quadratic equation for $H$.

We quantize the problem by considering a Schrödinger-like equation

$$
i \hbar \frac{\partial \psi(\vec{x}, t)}{\partial t}=\hat{H} \psi(\vec{x}, t)=\left[\hat{\vec{p}}^{2}-\frac{l^{2}}{r^{2}}+\frac{1}{r^{2}} \bar{l}^{2}\right] \Psi(\vec{x}, t)
$$

in which the operators $\hat{H}$ and $\hat{\vec{p}}$ are defined by the usual quantization rules

$$
\hat{H}=i \hbar \frac{\partial}{\partial t}, \quad \hat{p}_{l}=\frac{\hbar}{i} \partial_{l} .
$$

We see that the equation (3.3) describes a nonstandard form of a time dependent Schrödinger equation, with its right hand side containing both the first and second time derivatives (entering through $\bar{l}$ ).

For the stationary case, i.e. when $\Psi(\vec{x}, t)=\Psi_{E}(\vec{x}) e^{\frac{i E t}{\hbar}}$ we can use the angularmomentum basis and put

$$
\Psi_{E, m}=f_{E, m}(r) e^{i m \varphi}
$$

where $m$ is an integer, and find that $f_{E, m}$ satisfies a nonstandard time independent Schrödinger equation

$$
\left[-\hbar^{2}\left(\partial_{r}^{2}+\frac{1}{r} \partial_{r}-\frac{\bar{m}^{2}}{r^{2}}\right)-E\right] f_{E, m}(r)=0
$$

where we have defined

$$
\hbar \bar{m}:=\hbar m-\frac{\lambda}{4 \pi} E
$$

i.e. $\hbar \bar{m}$ is an eigenvalue of $\bar{l}$. 
A characteristic feature of the Schrödinger equation (3.6) is the appearance of the noncanonical angular momentum $\hbar \bar{m}$ with $\bar{m}$ not being an integer. i.e. our two-body system carries a fractional orbital angular momentum (see the discussion in [10] or [14]). It can be shown that $J=\bar{l}$ is equal to the total angular momentum of the particle + field system with a nonvanishing angular momentum of the fields [15].

In the following we discuss only the most interesting case of $\lambda \bar{l}<0$.

Now the appropriate boundary conditions correspond to the requirement that $f_{E, m}(r)$ is nonzero in either the interior region $\left(r<r_{0}\right)$ or in the exterior region $\left(r>r_{0}\right)$. Thus our boundary condition is

$$
f_{E, m}\left(r_{0}\right)=0
$$

The general solution of (3.6) is given by

$$
f_{E, m}(r)=Z_{\bar{m}}\left(\frac{\sqrt{E}}{\hbar} r\right),
$$

where $Z_{\bar{m}}$ is an appropriate Bessel function of order $\bar{m}$ (or a superposition of such functions).

\section{Interior solutions $\left(r<r_{0}\right)$}

The only Bessel functions belonging to a self-adjoint differential operator in (3.6) are those of the first kind with $\bar{m} \geq 0$. Therefore a decomposition of space into interior/exterior regions appear only in the case of $\lambda<0$.

Then the possible eigenvalues $E_{n}(m)$ are determined by

$$
J_{\bar{m}}\left[\frac{\sqrt{E}}{\hbar}\left(\frac{\hbar|\lambda| \bar{m}}{2 \pi}\right)^{\frac{1}{2}}\right]=0
$$

with $\bar{m}$ given by (3.7).

To simplify (3.10) we define

$$
\epsilon=\frac{|\lambda| E}{2 \pi \hbar}
$$

Then (3.10) takes the form

$$
J_{\bar{m}}\left(\bar{m}^{\frac{1}{2}} \epsilon^{\frac{1}{2}}\right)=0 .
$$

As $J_{\bar{m}}$, for fixed $\bar{m}>0$, has no imaginary zeros (see e.g. [16]) we have to consider an infinite number of simple positive zeros, which we denote by $y_{n}(\bar{m}), n=1,2$. arranged in ascending order of magnitude. Then we see that due to (3.7), the eigenvalues $\epsilon_{n}(m)$ we are looking for, are the positive fixed points of the equation

$$
\epsilon=f_{n}\left(m+\frac{1}{2} \epsilon\right)
$$


subject to the conditions $m+\frac{1}{2} \epsilon_{n}(m)>0$ and $y_{n}(\bar{m})>0$, where we have defined

$$
f_{n}(\bar{m}):=\frac{1}{\bar{m}} y_{n}^{2}(\bar{m})
$$

The solutions of (3.13) can be obtained only numerically.

We insert (3.14) into (3.13) and after the use of approximate formulae for the zeros of Bessel functions of first kind [16] we conclude that the low-lying energy levels are given by small values of $n$. In this case all values of $m \in \mathbb{Z}$ contribute to the energy spectrum $(E>0)$ but the positivity of $\bar{m}=m+\frac{1}{2} \epsilon_{n}(m)>0$ for higher $n$ implies that some finite range of values of $m$ has to be excluded. Qualitatively one can say that for every $n$ there appears however an infinite tower of energy values $\epsilon_{n}(m)$.

Only in the case of large $m$ the analytical asymptotic results are available. Let us consider the choice $n=1$. Using the asymptotic behaviour ([16])

$$
y_{1}(\bar{m})=\bar{m}+1.855757 \bar{m}^{\frac{1}{3}}+O\left(\bar{m}^{-\frac{1}{3}}\right),
$$

valid for large $\bar{m}$, we obtain

$$
\epsilon_{1}(m)=2 m+9.35243 m^{\frac{1}{3}}+O\left(m^{-\frac{1}{3}}\right) .
$$

valid for large positive $m$.

\section{Exterior solutions $\left(r>r_{0}\right)$}

First of all, let us note that there are no bound states solutions of (3.6) for $r>r_{0}$ as the only square integrable Bessel functions in $\left[r_{0}, \infty\right)$ are the modified Bessel functions of the third kind, which have neither positive nor pure imaginary zeros (see e.g. [16]).

Scattering solutions are given by a superposition of Bessel functions of the first and second kind

$$
f_{E, m}(r)=A_{m}(E) J_{\bar{m}}\left(\frac{\sqrt{E}}{\hbar} r\right)+B_{m}(E) Y_{\bar{m}}\left(\frac{\sqrt{E}}{\hbar} r\right)
$$

with the ratio $\frac{A_{m}}{B_{m}}$ determined by the boundary condition (3.8). These solutions describe scattering on an obstruction of radius $r_{0}$, which is dynamically determined.

\section{Final Remarks}

Gauging of translations for nonrelativistic point particles in a $D=2$ dimensional space coupled to a translational Chern-Simons action leads to a new and interesting $D=(2+1)$ particle dynamics. In particular, we have shown that for negative values of the product $\lambda \bar{l}$ of the coupling strength $\lambda$ and the fractional orbital angular momentum $\bar{l}$ the two-body motions separate into interior and exterior motions. In the quantum 
mechanical case we have obtained a new type of nonlinear Schrödinger equation, with a second order time derivative, which leads, in the stationary case, to the nonlinear energy eigenvalue problem. The interior solutions are described by a infinite tower of bound states for each fixed $n$. They describe confinement within a dynamically determined compact space region (see (2.41a)) (geometric bag). We should mention that analogous results were obtained by one of the present authors (PCS) in the onedimensional case [9]. It is clear that a possible physical relevance of these results, which recall the features of confinement in one and two space dimensions, should be investigated further.

\section{References}

[1] A. Staruszkiewicz, Acta Phys. Polon. 24, 734 (1963).

[2] S. Deser. R. Jackiw and G. 'tHooft, Ann. Phys. (NY) 152, 220 (1984)

[3] S. Deser, R. Jackiw and S. Templeton, Phys. Rev. Lett. 48, 975 (1982); Ann. Phys. (NY) 140,372 (1982).

[4] S. Carlip, "Quantum Gravity in 2+1 Dimensions", Cambridge Univ. Press 1998.

[5] E. Witten, Nucl. Phys. B311, 46 (1988).

[6] E.W. Mielke and P. Baeckler, Phys. Lett. 156A, 339 (1991).

[7] F. Tresquerres, Phys. Lett. 168A, 174 (1992).

[8] P.C. Stichel, Phys. Lett. 456B, 126 (1998).

[9] P.C. Stichel, "Gauging of 1d-space translations for nonrelativistic matter - geometric bags", hep-th/9911128.

[10] R. Jackiw and So-Young Pi, Phys. Rev. D 42, 3500 (1990).

[11] A. Khare, "Fractional Statistics and Chern-Simons Field Theory in $2+1$ Dimensions", hep-th/9908027.

[12] T. Regge and C. Teitelboim, Ann. Phys. 88, 286 (1974).

[13] P. Menotti and D. Seminara, "ADM Approach to 2+1 Dimensional Gravity", hep-th/9912263.

[14] R. Banerjee and B. Chakraborty, Phys. Rev. D49, 5431 (1994).

[15] J. Lukierski, P.C. Stichel and W.J. Zakrzewski, "From Gauging Nonrelativistic Translations to N-Body Dynamics", in preparation. 
[16] G.N. Watson, A Treatise on the Theory of Bessel Functions, Cambridge University Press, 1966. 